\title{
Author Index for Abstracts
}

Al-Kubati,M. 112 Alexander, K. 97,114 Anderson, E.-A. 113 Andersson, B. 103 Andersson, O.K. 103 Anger, H. 125 Antlsperger, A. 120 Assmann, G. 95 Augustin, H J. 123 Avramidis, P. 101

Backer, A. 94 Bähring, S. 107 Bajcetic, M. 113,122 Baldes, L. 101 Bandelow, N. 99 Barenbrock, M. 97,131,132 Barna,I. 128 Bartlomiejczyk, K. 130 Barton, M. 95 Bechstein, W.O. 119 Becker, R.H.A. 101 Berger, M. 122 Berlit, T. 93 Bethge,C. 121 Bias, A. 121 Binggeli,C. 115,116 Bischoff, A. 101 Block, D. 99,107 Bluemner, E.G. 130 Blum, U. 118 Bochmann, R.P. 133 Bohlender,J. 109,110 Böhm, M. 91,102 Bohmann, C. 93 Brehm, B.R. 96 Bremer, C. 117 Bretzel, R.G. 97 Bruneau, B.G. 104 Bubrov,L.L. 119 Busjahn, A. 110 Bychkov, R. 105,106 Carletti, M. 134 Chen, H. 94 Chen, J.H. 104 Chudek,J. 112 Chung, 0.110 Clancy, D.E. 123 Clozel, P.-J. 109 Compagnone, D. 124 Creutzig, A. 97,114 Culman, J. 100 Czycki, J. 105 d’Uscio, L.V 95 Dahmann, R. 96 Datté, J.-Y. 107 De Bald, A.J. 104 deChâtel,R. 128 de Matteo, C. 127 Deuber, H.J. 125 Dietze, G.J. 123 Drab, M. 106 Dudek, M. 133 Dudziak, M. 130 Dusek,J. 112

Ebner, S. 98 Eckert, S. 126 Eichhorn, J. 105 Eid,H. 104 Emden, J.C. 90 ErleyC. 127 Faulhaber, H.-D. 110 Feelisch, M. 96 Fischli, W. 109 Fiser,B. 112 Flachskampf, F.A. 134 Flaskamp,C. 134 Flesch, M. 102 Földes, K. 128 Francke, S. 91 Franke, A. 134 Franz, I.W. 124 Frei,U. 119 Frey, A.J.B. 99 Fritz, A. 118,134 Fürst, U. 101

Ganten,D. 109,110 Ganten,U. 110 Gasowski, J. 129 Gerö, L. 128 Ghiadoni, L. 117

Gleichmann, S. 126 Gleichmann, U. 126 Goeze, H. 127 Gohlke,P 107, 111 Golinski, P. 98 Gollasch, M. 105 Graf, K. 111 Granich, v. 124 Gretener, S. 116 Griebenow,R. 117,120

Gröbel, B. 125 Grodzicki, T. 129 Gross, V 106

Gryglewska, B. 129 Guckelberger, 0.119

Haller,H. 104,105,106,107 Handt, S. 108 Hanrath, P. 134 Haraldsson, B. 103 Hausberg,M. 113,114 Heinemann, L. 122,125 Heintz,B. 118,128,131,134 Heintze, U. 104,106 Heise, T. 122 Hellenkamp, A. 130 Heller, J. 94,100,102 Hempel, A. 104,106 Hendricks, P. 132 Henriksen, E.J. 123 Hense, H.-W132 Hermann, M. 104 Hessen, H.-C. 133 Heusser, K. 91 Heyl,W. 108,118,128 Hilgenfeldt,U. 111 Hilgers, K.F. 109 Hoffman, R.-P 113 Hohage, H. 93 Homuth, V105, 111 Horacek, V 100

Ivanov, M.A. 119 Ives,H.E. 106

Jacob, S. 123 Jandeleit-Dahm, K. 109 Jankowski, J. 92, 93, 96 Japundzic-Zigon, N. 113 Jee,W.S.S. 102 Johnston, C.I. 109 Jörger, U. 133 Jung, W.-1.123 Jungmann, E. 130 Kaiser, H.J. 131 Karsch, K.R. 96 Kehrel, B. 95 Keil,M. 101 Kelm, M.96,112,113 Kempler, P. 128 Khattab, M. 93 Kilter, H. 91 Kirch,W. 131 Kissel, S. 93 Kisters, K. 97, 99 Klaus, T. 97

Kling, H. 129

Knoblauch, H. 110

Koall, W. 125 
Kocemba, J. 129

Koch, A. 132

Koch,B. 102,121

Koch, K.M. 109

Kokot,F. 112

Kollenbaum, VE. 132

Kolloch, R.E. 91,133

Kramer, H.J. 94,100

Krampitz, G. 94

Krampitz, Jr. G. 94

Krebs, W. 134

Kresse, S. 125

Kristahn, K. 122

Krönig, B. 131

Krumme, B. 118

Krupa-Wojciechowska, B. 130,134

Kruse, H.-J. 97,114

Kubasik,A. 130

Kühl,H.P. 134

Kuroski de Bold, M.L. 104

Kuttler, B. 107

Lehmann, 122

Lehnert,T. 118

Lemmer,B. 103,118,128

Lennarz, M. 91,133

Lenz, O. 91

Li, J. 110

Lindschau, C. 104,105,106

Linz, W. 101,104

Lippert, T.H. 92,130

Lippoldt, A. 106

Liu, Y 122

Locher, R. 95

Lowe, S. 92

Ludwig, M. 91,133

Luft, F.C. 104,105,106,107,109,110

Lüscher, T.F. 95,114,115,116,127

Luther, H.P 111

Lydtin,H. 132

Maasch, C. 104 Magnusson, K. 125 Mann,H. 118 Marin-Grez, M.98,99 Mark, A.-L. 113

Matic, 1.122 Meier,B. 114,116,127 Ménard,J. 109,110 Mende, S. 90 Mentz, J. 134

Menz,M. 110 Meyer, C. 97 Meyer, G.J. 132 Meyer-Lehnert, H. 94 Michel, M.C. 94,101

Milau,C. 126 Mingers, S. 112 Montemurro, M. 90 Moreau, P. 95 Motz,W. 90,112

Mueck,A.O. 92, 130 Müller, D. 109 Müller,G.A. 104 Müller, J.F.M. 124 Münck,A.-C. 117

Murakami, K. 110 
Naraghi, R. 107 Naumann, H.-J. 133 Negrescu, E.V 97 Neuhaus, P. 119 Neusser, M. 98 Niedermayer, W. 132 Nofer, J.R. 95 Noll, G. 114,115,116 Nurminen, M.-L. 100

Obrezan,A.G. 119 Ogawa, T. 104 Olthoff, H. 105 Opitz-Gress, A. 122 Osten, B. 125 Ozóg, W. 129

Patschan, 0.107

Paul, M. 102

Pelemis, M. 122

Philipp, T. 94,117

Pietruck, F. 90

Pinto, Y 102

Pittrow, D.B. 120,131

Posadzy-Malaczynska, A. 127

Preik,M. 113

Prostran,M. 113,122

Prostran, M.S. 122

Radchenko, A. 124 Rahn, K.-H. 93, 99,132 Raszeja-Wanic, B. 127 Rath,W. 108,118,128,129

Reinecke, A. 111 Reister,F. 108,118 Rettig, R. 90, 99,107 Reusch, H.-P. 106 Reuter, U. 133

Ried, C. 105 Riehl,J. 134 Riester,U. 111 Risler, T. 127

Ritz,E. 111 Rosenfeld, S. 127 Rossberg, J. 132 Rosskopf, D. 90 Rott, A. 122 Rump, L.C.

93,118 Ruschitzka, F. 104, 129

Salvetti,A. 117

Samardzic, R. 113,122

Sandmann, S. 110

Sawicki,PT. 122,125

SchäferH. 110

Schäfer,S. 112,113

Schäfers, R.F. 94,117

Schardt, W. 120

Schiemann, A.L. 123

Schiffer, F. 102

Schindler, R. 119

Schlaich, M. 109

Schlatter, E. 93

Schlothmann, R. 97

Schlüter, H. 92, 93, 96, 98

Schmidt, W.E. 110

Schmieder, R.E. 91,109

Schnecko, A. 103

Schneider, W. 106

Schnepp, M. 125

Schnoor,P. 132

Schobel, H.R 91,109

Schölkens, B.A. 101

Schollmeyer, P. 93,118

Scholze, J. 123,124

Schöniger, R. 132 
Schölkens, B.A. 104

Schrader, J. 104,129

Schulz, E. 104,129

Schulze, K.J. 101

Schuster, H. 107,110

Schwertfeger, E. 93

Seeger, H.92, 130

Seibt,R. 133

Semetkowska-Jurkiewicz, E. 134

Shaw, S. 95

Sieberth, H.G. 118,131,134

SiegelovTsbá, J. 112

Siess, W. 97

Siffert, W. 90

Simon, 1.123

Sinkey,C.-A. 113

Sinz, V133

Sirenko, Y 124

Sitzler, G. 91

Skinner, S.-E. 95

SloniewskaM. 130

Smietanowski, M. 125

Smoleń, M. 129

Snelting, U. 130

Somers, V-K. 114

Spieker, C. 96, 97, 99,131,133 Spieker,L. 115,116 Spitznagel,H. 110 Stapff, M. 123

Stauch,M. 122 Stefanidis, I. 118 Steffen,H.M. 117 Steinacker, J.M. 122 Stevenson, M. 104

Stickan-Verfúrth, M. 101 Stimpel,M. 102,121 Strauer,B.E. 112,113 Stumpe, K.O. 91,113

Südkamp, M. 91 Sunder-Plassmann, L. 122 Suwelack, B. 132 Svedberg, J. 102 Sychev, O.

124

Taddei,S. 117

Tepel, M. 92,93, 95, 96, 97, 98 Theilmeier, G. 92 Thurau, K. 102 Todorovic, Z. 113,122

Toka, H. 107 Toka, 0.107 Tönnesmann, U. 124 Trenkwalder, P. 132 Trzebski, A. 125

Tschöpe, C. 111 Tykarski, A. 127

Uber, A. 107 Unger, Th. 100,110, 111

van Buuren, F. 126 Vanëcková, 1.102 Vasiljevic, Z. 122 Veelken,R. 109 Vereschagina, G.

120 Vetter, W. 95 Virdis,A. 117 Vogel, V 94 Vogt,M. 112 Völzke, H. 90

Wacker, P. 120 Wallukat,G. 111 Walter, M. 95, 97 Wambach,G. 94,117 Watson, S. 127

Weber, PC. 97 Weidinger,G. 120,131 Weihprecht, H. 109 Welzel,D. 120,131 Wenzel, R.R.

114,115,116,127 Wieçek,A. 112 Wieczorek, I. 97,114 Wieland,D. 118

Wiemer, G. 101 Wienker, T.F. 107 Will, D. 132 Willinek, W.A. 133 Wilson, E. 106

Wimberger, P. 99 Wimmer, E. 117 Winterberg, B. 131 Witta, J. 132 Witte,K. 103,118,128

Wobus, A. 106 Wurth, P. 118 Wysocki, M. 103

Yagil, C. 106 Yagil, Y 106 Yakhontov, D.A. 120 Yu,M.H. 111

Zapp,M.M. 131

Zbinden, S. 114 
Zdrojewski, T. 130

Zhu,YC. 110

Zhu,YZ. 110

Zhu, Z. 98

Zidek, W. 92, 93, 95, 96, 97, 99,131

Ziegler, A. 107

Ziegler, E.-M. 105

Zigon, N. 122

Zilles, P. 124

Züchner, C. 104

136

Author Index Vol. 20, No. 2, 1997 